\title{
Effects of macromolecular crowding on protein folding and aggregation
}

\section{Bert van den Berg, R.John Ellis ${ }^{1}$ and Christopher M.Dobson ${ }^{2}$}

Oxford Centre for Molecular Sciences, New Chemistry Laboratory, University of Oxford, South Parks Road, Oxford OX1 3QT and

${ }^{1}$ Department of Biological Sciences, University of Warwick, Coventry CV4 7AL, UK

${ }^{2}$ Corresponding author

e-mail: chris.dobson@chem.ox.ac.uk

We have studied the effects of polysaccharide and protein crowding agents on the refolding of oxidized and reduced hen lysozyme in order to test the prediction that association constants of interacting macromolecules in living cells are greatly increased by macromolecular crowding relative to their values in dilute solutions. We demonstrate that whereas refolding of oxidized lysozyme is hardly affected by crowding, correct refolding of the reduced protein is essentially abolished due to aggregation at high concentrations of crowding agents. The results show that the protein folding catalyst protein disulfide isomerase is particularly effective in preventing lysozyme aggregation under crowded conditions, suggesting that crowding enhances its chaperone activity. Our findings suggest that the effects of macromolecular crowding could have major implications for our understanding of how protein folding occurs inside cells.

Keywords: chaperone/hen lysozyme/macromolecular crowding/protein disulfide isomerase/protein folding

\section{Introduction}

The process of protein refolding in vitro has been studied extensively as a means of understanding how proteins fold inside cells. These experiments are, mainly for practical reasons, commonly carried out in simple buffer systems with low concentrations of protein in order to avoid aggregation during the refolding reaction. A major difference between these idealized conditions and those encountered within cells is that the intracellular environment is highly crowded due to the presence of high concentrations of soluble and insoluble macromolecules in the cytoplasm (Fulton, 1982; Minton, 1983; Zimmerman and Trach, 1991). Therefore, a significant fraction of the intracellular space is inaccessible to other macromolecules, and theory predicts that, as a result of this, macromolecular thermodynamic activities increase by several orders of magnitude (Minton, 1983; Zimmerman and Trach, 1991; Zimmerman and Minton, 1993). Therefore, biochemical rates and equilibria in a living cell may be quite different from those under idealized conditions (Zimmerman and Minton, 1993). Although the biophysical theory of macromolecular crowding is well developed (Minton, 1983,
1992; Minton et al., 1992), its biochemical consequences so far have hardly been tested (Ellis, 1997), the main exception being studies of DNA replication (Zimmerman and Minton, 1993). A qualitative prediction from crowding theory that could be relevant for protein folding in the cell is that macromolecular association constants under crowded conditions relative to those in dilute solution increase by as much as $2-3$ orders of magnitude, which, among other possible effects, could result in an increased propensity of folding protein molecules to aggregate (Zimmerman and Trach, 1991; Zimmerman and Minton, 1993; Ellis and Hartl, 1996). It is therefore surprising that the effects of macromolecular crowding on protein refolding have been neglected, with the exception of investigations of its significance for GroEL-mediated protein refolding (Burston et al., 1996; Martin and Hartl, 1997).

Here we describe the effects of polysaccharide and protein macromolecular crowding agents on protein refolding and aggregation. Lysozyme is a particularly suitable system for this purpose, since its refolding has been studied in detail both under conditions where the native disulfide bonds are maintained throughout the refolding reaction and under oxidative conditions (Chaffotte et al., 1992; Radford et al., 1992; Dobson et al., 1994; Roux et al., 1997; van den Berg et al., 1999a,b). In contrast to the oxidized protein, the reduced protein is known to be highly susceptible to aggregation (Goldberg et al., 1991). This makes it possible to compare the effects of crowding on the same polypeptide chain in both aggregation-prone and aggregation-resistant states.

The results of this study show that the yield of correctly folded enzyme produced from reduced lysozyme decreases dramatically at concentrations of four different crowding agents comparable to macromolecule concentrations present in cells. The decreased refolding yields under crowded conditions do not result from slower refolding rates as a possible consequence of the increased viscosity of the refolding buffers, since the addition of crowding agents accelerates the refolding process. The effects of the protein crowding agents are even more pronounced than those of the polysaccharides, suggesting that, in addition to excluded volume effects, protein-protein interactions can also contribute to the decreased refolding yields. Protein concentration and light scattering measurements demonstrate that the decreased refolding yields of the reduced protein under crowded conditions are due to aggregation. In contrast, the refolding of the protein containing the native disulfide bonds is not affected by crowding. Since the rate of refolding of the reduced protein is $\sim 2-3$ orders of magnitude slower than that of the protein with the disulfide bonds intact, the results suggest that folding rate is an important determinant of whether a protein is susceptible to aggregation. In accord 


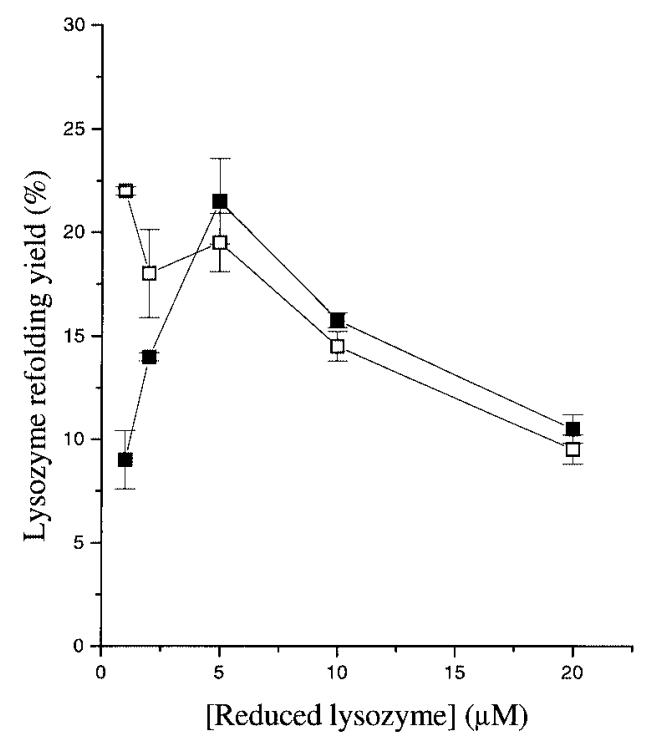

Fig. 1. Refolding yields as a function of lysozyme concentration. Reduced lysozyme was refolded in the absence $(\boldsymbol{\square})$ or presence $(\square)$ of $1 \mathrm{mg} / \mathrm{ml} \mathrm{BSA}$, in the presence of 2.0/1.0 $\mathrm{mM}$ reduced/oxidized glutathione at $\mathrm{pH} 7.4$ and $37^{\circ} \mathrm{C}$.

with this, the action of the protein folding catalyst protein disulfide isomerase (PDI) results in the rescue from aggregation of a proportion of the lysozyme molecules both in the presence and absence of crowding agents. The observation that the refolding rates are relatively constant at higher concentrations of PDI, in combination with its known abundance within the endoplasmic reticulum (ER) (Hillson et al., 1984; Zapun et al., 1992), suggest, however, that at higher concentrations of PDI its chaperone activity is the dominant factor in preventing aggregation of refolding lysozyme. PDI is particularly effective in preventing lysozyme aggregation under crowded conditions, suggesting that crowding enhances its chaperone function.

\section{Results and discussion}

\section{Refolding yield as a function of lysozyme concentration}

It has been shown previously that, after initiation of the refolding of reduced lysozyme, a rapid partitioning occurs between species committed to productive folding and species committed to aggregation, which results in rapidly decreasing refolding yields at higher protein concentrations (Goldberg et al., 1991). Although reduced lysozyme can be refolded efficiently in the presence of sufficiently high concentrations of denaturants (van den Berg et al., 1999a,b), we chose to refold the reduced protein in the absence of denaturants in the present work in order to mimic more closely the conditions relevant to the folding of a nascent polypeptide chain in vivo. This was done by using a protocol in which the denatured protein was solubilized in dilute acid prior to dilution (see Materials and methods). Figure 1 shows the refolding of reduced lysozyme using this protocol as a function of its concentration at $\mathrm{pH} 7.4$ and $37^{\circ} \mathrm{C}$ as measured by the recovery of enzymatic activity, which is dependent on the formation of the correct disulfide bonds in lysozyme. Concentrations of 2.0/1.0 mM were chosen for reduced/oxidized glutathione since these result in optimal refolding yields (data not shown). These concentrations are similar to those present in the secretory pathway (Hwang et al., 1992), although the mechanism of oxidation of cellular protein thiols may involve other factors (Cuozzo and Kaiser, 1999). The results confirm that reduced lysozyme is prone to aggregation during its refolding; at an optimal lysozyme concentration of $5 \mu \mathrm{M}$, the refolding yields are only $\sim 20$ $25 \%$. Allowing for different experimental conditions such as $\mathrm{pH}$ and temperature, this yield is in reasonable agreement with previously reported data (Goldberg et al., 1991; Puig and Gilbert, 1994). At concentrations both lower and higher than $5 \mu \mathrm{M}$ lysozyme, the refolding yields decrease. The decrease at higher lysozyme concentrations is expected for an aggregation-prone protein, whereas the decrease in refolding yield at low lysozyme concentrations most probably reflects non-specific absorption of protein on the Eppendorf vial surface, which can be overcome by addition of low concentrations of bovine serum albumin (BSA) (Puig and Gilbert, 1994). As Figure 1 shows, at lysozyme concentrations $\geqslant 5 \mu \mathrm{M}$, the contribution of nonspecific absorption is negligible.

\section{Effects of macromolecular crowding agents on aggregation during lysozyme refolding}

The synthetic polysaccharides Ficoll 70 and dextran 70 are well suited as background species (Zimmerman and Minton, 1993) to mimic the intracellular crowded environment in vitro, since they are inert, highly soluble and have an average molecular mass of $\sim 70 \mathrm{kDa}$. In addition to these compounds, it was decided to use BSA and chicken albumin (ovalbumin) as crowding agents, in order to monitor any effects due to more specific interactions of these proteins with refolding lysozyme. The results are summarized in Figure 2A. It is clear that the addition of all crowding agents at high concentrations prevents correct refolding of reduced lysozyme. Interestingly, the effects of the non-interacting crowding agents Ficoll 70 and dextran 70 on lysozyme refolding are similar, although dextran 70 has a more asymmetrical structure and therefore a substantially higher viscosity than Ficoll solutions of the same concentration (Martin and Hartl, 1997). This observation strongly suggests that the decrease in refolding yield in the presence of the crowding agents is not due to a higher viscosity of the solutions and hence to a slower rate of refolding. This notion is confirmed by the results shown in Figure 2B, where the yields and rates of refolding are shown in the presence and absence of $80 \mathrm{~g} / 1$ Ficoll. At this concentration of Ficoll, the refolding yields are substantially lower than those obtained in the absence of crowding agents, but still high enough to allow us to measure the rate of the refolding process accurately. Strikingly, the observed refolding rate is significantly faster in the presence of Ficoll. This observation demonstrates that the increased viscosity of solutions containing Ficoll does not contribute to the decrease in refolding yields and, importantly, suggests that crowding can actually enhance rates of protein refolding.

Figure 2A shows that there is a marked difference between the action of the polysaccharide and protein crowding agents. The yields of correctly folded lysozyme are lower in the presence of protein crowding agents compared with those obtained at the same concentrations of polysaccharide crowding agents. Although during the 
A

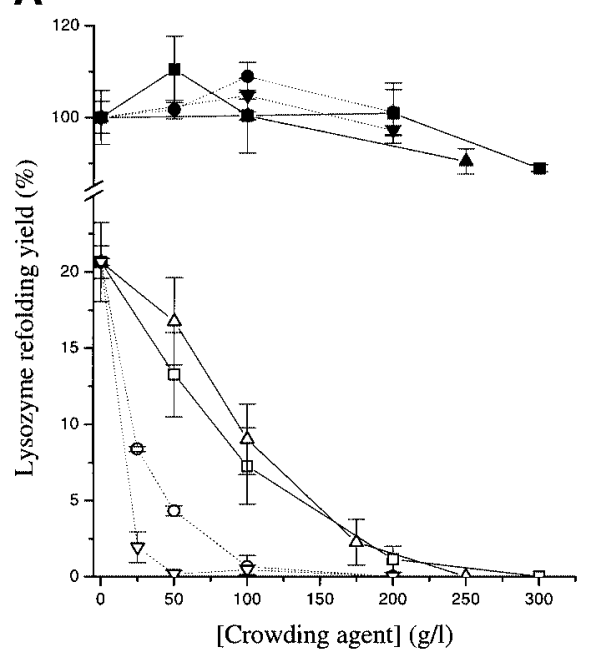

B

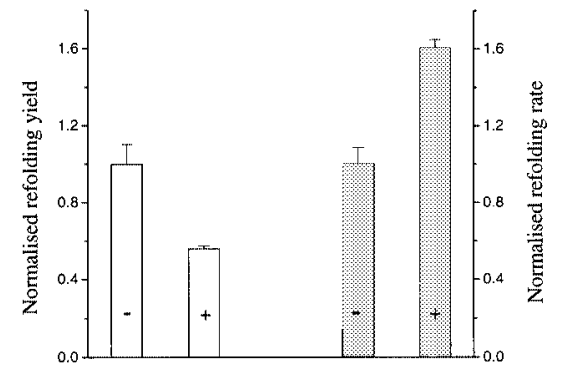

Fig. 2. Effects of macromolecular crowding agents on lysozyme refolding. (A) Reduced (open symbols) or oxidized lysozyme (refolded from $8 \mathrm{M}$ urea; closed symbols) was refolded in the presence of varying concentrations of Ficoll $70(\boldsymbol{\square}, \square)$, dextran $70(\boldsymbol{\Lambda}, \triangle)$, BSA $(\boldsymbol{O}, \bigcirc)$ and ovalbumin $(\boldsymbol{\nabla}, \nabla)$. (B) Normalized refolding yields (white bars) and observed refolding rates (grey bars) obtained by refolding reduced lysozyme in the absence $(-)$ and presence $(+)$ of $80 \mathrm{~g} / 1$ Ficoll 70. Lysozyme $(5 \mu \mathrm{M})$ was refolded in the presence of $2.0 /$ $1.0 \mathrm{mM}$ reduced/oxidized glutathione, at $\mathrm{pH} 7.4$ and $37^{\circ} \mathrm{C}$.

refolding process lysozyme molecules are likely to experience somewhat different excluded volume interactions with a globular protein such as BSA compared with uncross linked or partially cross linked polysaccharide chains of similar molecular weight (dextran 70), this observation suggests that additional factors besides size exclusion effects play a role in determining protein refolding yields. The exact nature of any interactions of lysozyme with BSA and ovalbumin is unknown, but the fact that hen lysozyme is a highly basic protein with an isoelectric point (pI) of 11.0 makes it likely to interact electrostatically with acidic proteins such as BSA and ovalbumin (which have pIs of 4.6 and 4.9 , respectively) at the $\mathrm{pH}$ of 7.4 used here. Moreover, since BSA and ovalbumin both contain a single free cysteine residue, intermolecular disulfide bonds could be formed between these protein crowding agents and lysozyme refolding intermediates, resulting in lower refolding yields. In order to ensure that the observed decreases in lysozyme refolding yields are not caused by inhibitory effects of the crowding agents on lysozyme itself or on the enzymatic activity assay, native lysozyme was incubated for up to $48 \mathrm{~h}$ with the highest concentrations of crowding agents used in this study. Subsequent analysis of the specific enzymatic activities of these samples showed that these were identical to the specific activity of native lysozyme, excluding any inhibitory effects by the crowding agents on lysozyme or the enzymatic activity assay.

When oxidized lysozyme is refolded from $8 \mathrm{M}$ urea in the presence of crowding agents, the yields of correctly folded protein are hardly affected. Identical results were obtained by refolding from $6 \mathrm{M}$ guanidine $\mathrm{HCl}(\mathrm{GuHCl})$ as a denaturant (data not shown). Only at concentrations of crowding agents in excess of $250 \mathrm{~g} / \mathrm{l}$ does the refolding yield decrease slightly to $90 \%$ (Figure 2A). It could be argued that the lack of effects of the crowding agents on oxidized lysozyme is due to the presence of residual low concentrations of denaturants $(160 \mathrm{mM}$ urea or $120 \mathrm{mM}$ $\mathrm{GuHCl}$ ) during its refolding (see Materials and methods). However, when reduced lysozyme was refolded from $8 \mathrm{M}$ urea or $6 \mathrm{M} \mathrm{GuHCl}$ under otherwise identical conditions, very similar refolding yields were obtained compared with those found when reduced lysozyme is refolded from acid in the absence of these denaturants (data not shown). This observation excludes prevention of aggregation by low concentrations of denaturants as an explanation for the absence of crowding effects during refolding of oxidized lysozyme, and demonstrates that the refolding yields of reduced lysozyme do not depend on the conditions from which the protein was refolded in the present study.

Since crowding theory predicts that aggregation should increase under conditions of macromolecular crowding, it is important to demonstrate that the observed decreases in lysozyme yield under crowded conditions as measured by enzymatic activity are actually caused by aggregation. The data shown in Figure 3A show that this is the case, since the observed light scattering of samples refolded in the presence of $200 \mathrm{~g} / \mathrm{l}$ Ficoll 70 or dextran 70 is substantially higher than that of those refolded without crowding agents. The fact that some scattering is also observed in the samples refolded in the absence of crowding agents reflects the propensity of reduced lysozyme to aggregate even in the absence of crowding agents, and is compatible with the enzymatic activity data (Figure 2A). When samples are centrifuged after refolding, and the concentration of soluble protein determined by absorbance at $280 \mathrm{~nm}$, a good correlation is obtained with the enzymatic activity and light scattering data (Figure 3B). Comparison of the refolding yields in Figures $2 \mathrm{~A}$ and $3 \mathrm{~B}$ demonstrates that the aggregates that are formed are large, and that most, though not all, of the protein remaining in solution after centrifugation corresponds to correctly folded native lysozyme. The amount of scattering observed in samples containing protein crowding agents is less than in those containing polysaccharide crowding agents (Figure 3A). This is especially true in the case of BSA, and could indicate that the nature of the lysozyme aggregates formed in the presence of the protein crowding agents is different from those formed in the presence of the polysaccharide crowding agents. As mentioned above, it is also possible that some soluble complexes between BSA and lysozyme refolding intermediates could have been formed as a result of more specific protein-protein interactions.

\section{PDI-assisted lysozyme refolding under crowded conditions}

The refolding of oxidized lysozyme is fast and essentially complete after a few seconds, excluding a minor population 

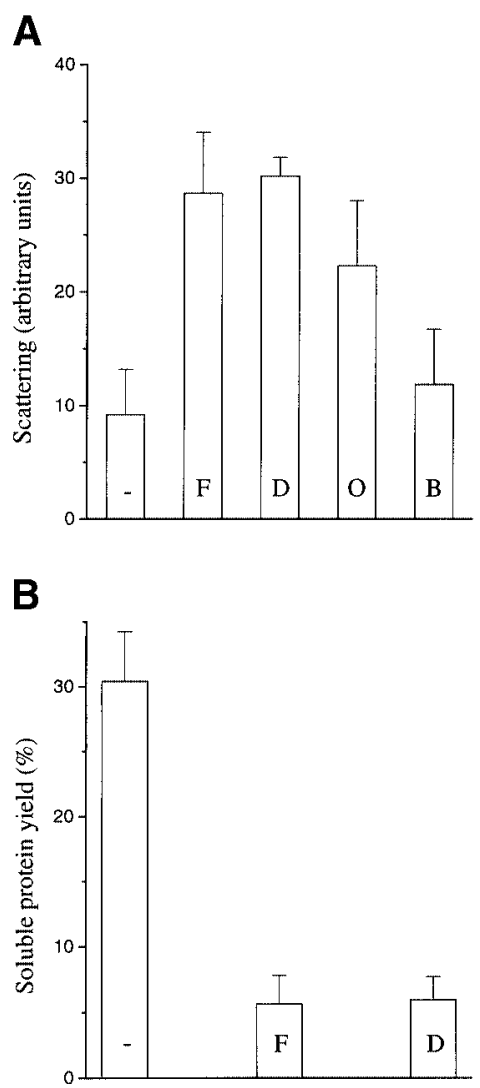

Fig. 3. Decreased refolding yields under crowded conditions are caused by aggregation. (A) Aggregation of lysozyme refolding samples determined in the absence $(-)$ and presence of macromolecular crowding agents (F, 200 g/l Ficoll 70; D, 200 g/l dextran 70; O, $100 \mathrm{~g} / 1$ ovalbumin; B, $100 \mathrm{~g} / \mathrm{l} \mathrm{BSA})$. Aggregation was measured by fluorescence emission at $455 \mathrm{~nm}$ after $6 \mathrm{~h}$ of refolding. (B) Measurement of soluble protein yield by absorption at $280 \mathrm{~nm}$ following centrifugation of lysozyme samples refolded for $6 \mathrm{~h}$ in the absence (-) and presence of crowding agents (F, 200 g/l Ficoll 70; D, $200 \mathrm{~g} / \mathrm{l}$ dextran 70$)$. Reduced lysozyme $(5 \mu \mathrm{M})$ was refolded in the presence of $2.0 / 1.0 \mathrm{mM}$ reduced/oxidized glutathione, at $\mathrm{pH} 7.4$ and $37^{\circ} \mathrm{C}$.

that folds more slowly [half-time of folding $(\tau) \sim 15-20 \mathrm{~s}$ ], perhaps as a result of cis-trans prolyl isomerization (Radford et al., 1992; Dobson et al., 1994). In contrast, the oxidative refolding of the reduced protein is $2-3$ orders of magnitude slower as the rate is limited by the need to form the native-like disulfide bonds; $\tau \sim 15-20$ min under these conditions, as the cysteine residues are predominantly uncharged at pH 7.4 (Weissman and Kim, 1991). Since it is known that, during non-oxidative folding, intermediates with exposed hydrophobic surfaces are extensively populated (Radford et al., 1992; Dobson et al., 1994), the absence of large effects of crowding on the refolding of the oxidized protein is likely to be caused by the high rate of this process compared with the much slower refolding of the reduced protein. It is therefore of interest to study the effect of PDI, the major catalyst of disulfide bond formation present within the ER (Freedman et al., 1994; Gilbert, 1997), on the yields and rates of the refolding of the reduced protein. The results are summarized in Figure 4.

It is clear that addition of PDI leads to a large increase in the refolding yield of lysozyme in the absence of crowding agents (Figure 4A); in the presence of $20 \mu \mathrm{M}$
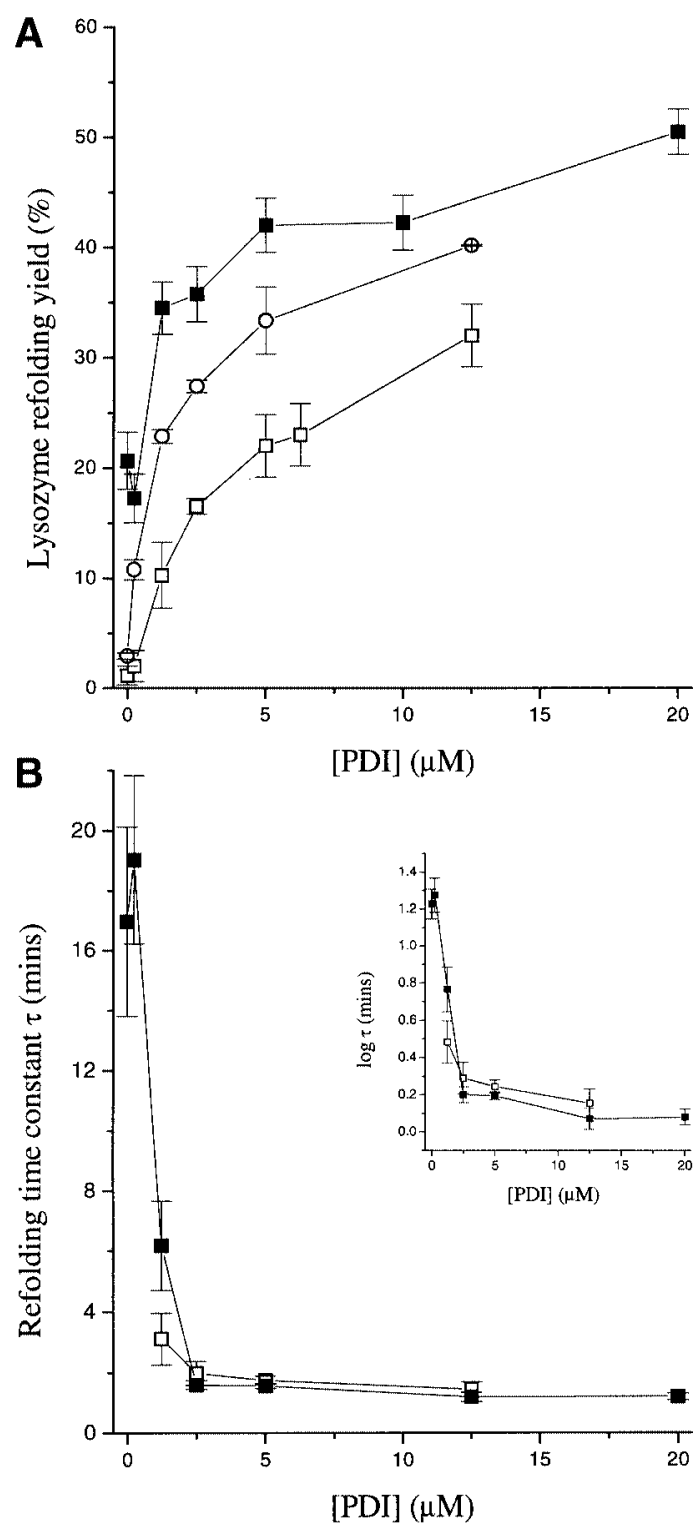

Fig. 4. PDI prevents aggregation of refolding lysozyme under conditions of macromolecular crowding. (A) Effects of PDI on the refolding yield of lysozyme in the absence of crowding agents $(\boldsymbol{\square})$ and in the presence of $200 \mathrm{~g} / \mathrm{l} \mathrm{Ficoll}(\square)$ and $100 \mathrm{~g} / \mathrm{l} \mathrm{BSA}(\bigcirc)$. (B) Time constants of lysozyme refolding $(\tau)$ obtained at various PDI concentrations in the absence of crowding agents (ם) and in the presence of $200 \mathrm{~g} / \mathrm{l}$ Ficoll $(\square)$. The inset to $(B)$ shows $\log (\tau)$ versus PDI concentration. Lysozyme refolding rates in the presence of Ficoll could not be determined at very low concentrations of PDI due to the low yields of the refolding reaction. Refolding was carried out with $5 \mu \mathrm{M}$ reduced lysozyme at $\mathrm{pH} 7.4$ and $37^{\circ} \mathrm{C}$.

PDI, refolding yields are $\sim 50 \%$, compared with only $\sim 20 \%$ in the absence of PDI. The effect of PDI on lysozyme refolding yields is relatively even more pronounced in the presence of crowding agents. In the presence of both Ficoll and BSA, the lysozyme refolding yields increase from $<3 \%$ in the absence of PDI to $30-40 \%$ at the highest concentration of PDI investigated $(12.5 \mu \mathrm{M})$. Similar results were obtained with dextran 70 and ovalbumin as crowding agents (data not shown). In contrast to results obtained previously for bovine PDI (Puig and Gilbert; 1994; Puig et al., 1994), no evidence of significant antichaperone activity of human PDI was found in this or our 
earlier studies (van den Berg et al., 1999b). Human PDI present at substoichiometric concentrations relative to lysozyme markedly increases lysozyme refolding yields in both the presence and absence of crowding agents (Figure 4A). The fact that the human protein is already effective at low concentrations in improving lysozyme refolding yields suggests that at least part of this effect can be attributed to the rate of acceleration by PDI of lysozyme refolding.

In Figure 4B, the time constants of lysozyme refolding in the absence and presence of $200 \mathrm{~g} / \mathrm{l}$ Ficoll are shown as a function of the PDI concentration. It is clear that, in the absence of the crowding agent, addition of $2.5 \mu \mathrm{M}$ PDI results in a large decrease ( 7-fold) in the time constant for the refolding process under these conditions. However, as has been reported previously (Puig and Gilbert, 1994), the rates of the PDI-assisted refolding process are relatively constant $(\tau \sim 1.5 \mathrm{~min})$ at higher PDI concentrations, whereas the refolding yields as shown in Figure 4A continue to increase up to at least $20 \mu \mathrm{M}$ PDI. It is therefore tempting to speculate that the increase in lysozyme refolding yields resulting from the addition of PDI is due to an increase in the refolding rates at low concentrations of PDI, but that at higher concentrations of PDI its chaperone function (Puig and Gilbert, 1994; Song and Wang, 1995; Gilbert, 1997) becomes important in increasing the yield of the refolding process. From the yield of PDI purified from liver (Hillson et al., 1984), and assuming that the ER lumen represents $\sim 10 \%$ of the cell volume, it can be estimated that the concentration of PDI in vivo is $\sim 200 \mu \mathrm{M}$. Such a high concentration could exert a strong chaperone effect in reducing aggregation.

Due to the low refolding yields in the presence of high concentrations of Ficoll, it is not possible to determine refolding rates under these conditions in the absence of PDI, or when it is present only at very low concentrations. However, at higher PDI concentrations $(2.5-12.5 \mu \mathrm{M})$, the refolding rates are very similar in $200 \mathrm{~g} / \mathrm{l}$ Ficoll compared with those in the absence of Ficoll (Figure 4B). Moreover, Figure 4A shows that over this range of PDI concentrations the refolding yields in the absence of Ficoll increase only by $\sim 20 \%$, whereas in the presence of Ficoll the increase in refolding yield is $\sim 2$-fold. Since the refolding rates at higher PDI concentrations are relatively constant and very similar in the presence and absence of crowding agents, this observation suggests that PDI is a more effective chaperone under crowded conditions. This suggestion is in excellent agreement with the higher association constants of interacting macromolecules predicted by crowding theory (Zimmerman and Minton, 1993).

\section{Implications for studies of protein refolding}

The present study is consistent with the prediction that excluded volume effects resulting from macromolecular crowding increase association constants, leading to increased aggregation during the refolding of reduced lysozyme. Although no other protein systems have been investigated in the present study, it is likely that macromolecular crowding will affect the folding and aggregation of a subset of cellular proteins. We propose this because different proteins vary greatly in their tendency to aggregate during refolding, and our results suggest that crowding will affect mainly those proteins that are prone to aggregation, either because they fold slowly or because they fold through significantly populated aggregationprone intermediate states.

Evidence that macromolecular crowding is an actual intracellular phenomenon comes from measurements of protein diffusion rates in the cytoplasm of both eukaryotic and prokaryotic cells. The reduction in translational and rotational diffusion rates of green fluorescent protein (GFP) expressed in the cytoplasm of Chinese hamster ovary $(\mathrm{CHO})$ cells is consistent with protein crowding equivalent to $\sim 80-120 \mathrm{~g} / \mathrm{l}$ of dextran (Swaminathan et al., 1997). Recent results suggest that diffusion rates in the cytoplasm of Escherichia coli are also compatible with those expected to result from highly crowded conditions as a result of protein concentrations in the order of 200$300 \mathrm{~g} / \mathrm{l}$ (Elowitz et al., 1999). Although no reliable estimates have been reported for the total protein concentration within the lumen of the ER, the general consensus is that it is very high and hence that the ER is a crowded environment. Thus crowding potentially will affect the folding of a large number of proteins, both in the cytoplasm and in the ER lumen.

All these considerations suggest that strategies must exist to combat the effects of crowding that reduce the potential yield of correctly folded proteins. One of these strategies, the reduction of the diffusion of macromolecules, is a direct effect of macromolecular crowding (Minton et al., 1982; Zimmerman and Minton, 1993). In addition, the observation that relatively low concentrations of crowding agents can enhance refolding rates significantly in vitro (Figure 2B) suggests that proteins might fold more quickly in vivo as a result of crowding, consequently making them less susceptible to aggregation. A strategy that the cell has developed during evolution in order to avoid aggregation of folding polypeptide chains is to employ molecular chaperones (Ellis, 1997), which essentially reduce aggregation either by lowering the effective concentration of nascent and newly synthesized polypeptide chains by binding to them (Hartl, 1996) or by allowing some chains to fold in a sequestered microenvironment in the case of chaperonins (Ellis and Hartl, 1999). Both these mechanisms are likely to be facilitated by crowding, since this phenomenon should also increase association between chaperones and their target polypeptides (Figure 4; Martin and Hartl, 1997).

The large effects of protein crowding agents on lysozyme refolding (Figure 2A) suggest that, in addition to non-specific excluded volume effects, more specific protein-protein interactions could greatly affect protein folding in the highly complex intracellular environment, where large numbers of proteins with very different properties are present. Since these protein-protein interactions are also likely to be enhanced by macromolecular crowding, these findings stress the importance of strategies that the cell must employ in order to minimize the interactions of refolding proteins not only with themselves, but also with other proteins present in the cell.

Although at present it is not fully established which crowding agents simulate the intracellular environment most accurately, the fact that similar results are obtained by using crowding agents with very different physicochemical properties suggests that the qualitative effects of macro- 
molecular crowding are not critically dependent on the nature of the crowding agent employed. Moreover, the effects described in this study are observed with concentrations of crowding agents that are in the same general range as macromolecule concentrations found in the cytoplasm (80-200 g/l; Swaminathan et al., 1997).

The dramatic effects of crowding agents on lysozyme aggregation during refolding, together with the effectiveness of PDI in improving lysozyme refolding yields under crowded conditions, suggest that the increase in macromolecular association constants caused by macromolecular crowding could influence many aspects of protein folding processes. Moreover, our observation that protein refolding rates can be faster in the presence of crowding agents is consistent with another prediction of macromolecular crowding theory which postulates that compact, globular states are favoured over asymmetric ones (Zimmerman and Minton, 1993), and could imply that crowding accelerates intrinsic rates of protein folding.

The present work on protein refolding complements that of an earlier study of the effect of high concentrations of inert macromolecules on the process of protein unfolding (Minton et al., 1982). In this earlier study, it was found that the rate and extent of aggregation of several proteins denatured by heat or ethanol were reduced by the addition of other stable proteins. The effects of crowding may, therefore, in some cases be of advantage to the cell as well as detrimental. Further studies of macromolecular crowding should therefore contribute significantly to our understanding of the nature of protein aggregation processes and of the mechanisms that have evolved to overcome the problems these could potentially cause in a cellular environment.

\section{Materials and methods}

\section{Reagents}

HEPES and urea were obtained from Sigma; $\mathrm{KCl}, \mathrm{MgCl}_{2}$, potassium phosphate and glucose were from BDH Laboratory supplies. Hen lysozyme was obtained from Sigma and used without further purification. BSA (essentially fatty acid free), chicken albumin (ovalbumin; grade V) and Ficoll 70 were obtained from Sigma. Dextran T70 was obtained from Pharmacia. Human PDI was produced in E.coli strain Bl21(DE3) (Darby et al., 1998), and was a generous gift from Dr Johan Kemmink (University of Canterbury, UK). PDI concentrations (expressed as monomers) were determined using an $E_{1 \%}$ at $280 \mathrm{~nm}$ of 0.9 .

\section{Reduction and renaturation of lysozyme}

Lysozyme was reduced in the presence of dithiothreitol (DTT) and high concentrations of denaturants as previously described (van den Berg et al., 1999a). Lyophilized aliquots of reduced protein $(100 \mu \mathrm{M})$ were dissolved in acetic acid $\mathrm{pH} 3$ immediately before use. In control experiments, aliquots of the reduced protein were dissolved at $250 \mu \mathrm{M}$ in refolding buffer containing $8 \mathrm{M}$ urea or $6 \mathrm{M} \mathrm{GuHCl}$. Refolding of the reduced protein was initiated by depositing a droplet of a concentrated solution on the wall of the Eppendorf tube followed by rapid dilution into the refolding buffer, effected by vigorous agitation with a Vortex mixer for $3 \mathrm{~s}$. A similar procedure has been shown previously to result in reproducible mixing conditions (Goldberg et al., 1991). The refolding buffer was optimized in order to mimic physiological conditions closely and to give the highest refolding yields. The optimal refolding buffer consisted of $100 \mathrm{mM}$ HEPES, $100 \mathrm{mM} \mathrm{KCl,} 5 \mathrm{mM} \mathrm{MgCl} 2 \mathrm{pH}$ 7.5. Stock solutions of crowding agents (200-350 g/l) were made in refolding buffer. Stock solutions of reduced and oxidized glutathione in water $(100 / 50 \mathrm{mM}$, respectively) were made immediately prior to use and added to the refolding buffer before the addition of lysozyme. Final concentrations of reduced/oxidized glutathione in the refolding experiments were 2.0/1.0 mM, respectively. For experiments involving PDI, reduced lysozyme was added to refolding solutions already containing
PDI. After addition and mixing of all components, the final $\mathrm{pH}$ value in the refolding experiments was $7.40 \pm 0.05$. All refolding experiments were carried out at $37^{\circ} \mathrm{C}$. The reported refolding yields are averages of at least three different experiments with different protein samples. Time constants $(\tau)$ are defined as the half-times of lysozyme refolding, and were determined by fitting the increase in enzymatic activity versus time to a single exponential model (van den Berg et al., 1999a).

\section{Refolding of oxidized lysozyme}

Initiation of refolding of disulfide-intact lysozyme was carried out in an analogous way to that described above, by a 50-fold dilution of stock solutions of $250 \mu \mathrm{M}$ denatured lysozyme into refolding buffer containing $8 \mathrm{M}$ urea or $6 \mathrm{M} \mathrm{GuHCl}$, resulting in final concentrations of $160 \mathrm{mM}$ urea or $120 \mathrm{mM} \mathrm{GuHCl}$ in the refolding buffer.

\section{Lysozyme activity assay}

Refolding samples of reduced and oxidized lysozyme were assayed after $4-6 \mathrm{~h}$ of refolding. Control experiments assayed after 24 and $48 \mathrm{~h}$ showed that the enzymatic activities of the refolding samples did not increase further after $4 \mathrm{~h}$ of refolding. Enzymatic activities were assayed by mixing an aliquot of lysozyme with $1 \mathrm{ml}$ of a Micrococcus lysodeikticus cell wall suspension of OD 0.6-1.0 in $50 \mathrm{mM}$ potassium phosphate, $0.1 \%$ glucose at $\mathrm{pH} 7.4$, and monitoring the decrease in absorbance at $450 \mathrm{~nm}$ during $30 \mathrm{~s}$. All enzymatic activities were normalized against and expressed as a percentage of a $5 \mu \mathrm{M}$ solution of native lysozyme in refolding buffer. Control experiments were performed to ensure that the crowding agents did not inhibit native lysozyme or have any influence on the activity assay. Each sample was assayed at least three times since the error in these measurements is $\pm 5-10 \%$.

\section{Measurements of protein aggregation}

Refolding samples in the absence and presence of crowding agents were transferred to quartz cuvettes after $6 \mathrm{~h}$ of refolding and their fluorescence measured on a Perkin Elmer LS 50B luminescence spectrometer. An excitation wavelength of $450 \mathrm{~nm}$ was used with an emission wavelength of $455 \mathrm{~nm}$ (emission/excitation slit widths $2.5 / 2.5 \mathrm{~nm}$ ), in order to measure light scattering. Measuring the absorbance at $450 \mathrm{~nm}$ gave qualitatively similar results (data not shown). Subsequently, samples were centrifuged for $45 \mathrm{~min}$ on a Jouan A-14 table centrifuge operating at 14000 r.p.m., and the absorbance at $280 \mathrm{~nm}$ was measured in order to determine the amount of soluble protein remaining in solution. The percentage values reported in Figure $3 \mathrm{~B}$ were obtained by normalization against the absorbance of a $5 \mu \mathrm{M}$ solution of native lysozyme $(100 \%$ refolding yield) in the respective buffers. In all experiments, blanks were subtracted in order to correct for scattering and absorbance of buffer components (including crowding agents).

\section{Acknowledgements}

We would like to thank Johan Kemmink (University of Canterbury, UK) for his generous gift of recombinant human PDI. This is a contribution from the Oxford Centre for Molecular Sciences, which is supported jointly by the BBSRC, EPSRC and MRC. B.v.d.B. is supported by an EU grant. The research by C.M.D. is supported in part by the Howard Hughes Medical Institute and by the Wellcome Trust.

\section{References}

Burston,S.G., Weissman,J.S., Farr,G.W., Fenton,W.A. and Horwich,A.L. (1996) Release of both native and non-native proteins from a cis-only GroEL ternary complex. Nature, 383, 96-99.

Chaffotte,A.-F., Guillou,Y. and Goldberg,M.E. (1992) Kinetic resolution of peptide bond and side chain far-UV circular dichroism during the folding of hen egg white lysozyme. Biochemistry, 31, 9694-9702.

Cuozzo,J.W. and Kaiser,C.A. (1999) Competition between glutathione and protein thiols for disulfide-bond formation. Nature Cell Biol., 1, 130-135.

Darby,N.J., Penka,E. and Vincentelli,R. (1998) The multi-domain structure of protein disulfide isomerase is essential for high catalytic activity. J. Mol. Biol., 276, 239-247.

Dobson,C.M., Evans,P.A. and Radford,S.E. (1994) Understanding how proteins fold: the lysozyme story so far. Trends Biochem. Sci., 19, 31-37.

Ellis,R.J. (1997) Molecular chaperones: avoiding the crowd. Curr. Biol., 7, R531-R533.

Ellis,R.J. and Hartl,F.-U. (1996) Protein folding in the cell: competing models of chaperonin function. FASEB J., 10, 20-26. 
Ellis,R.J. and Hartl,F.-U. (1999) Principles of protein folding in the cellular environment. Curr. Opin. Struct. Biol., 9, 102-110.

Elowitz,M.B., Surette,M.G., Wolf,P.-E., Stock,J.B. and Leibler,S. (1999) Protein mobility in the cytoplasm of Escherichia coli. J. Bacteriol., 181, 197-203.

Freedman,R.B., Hirst,T.R. and Tuite,M.F. (1994) Protein disulphide isomerase: building bridges in protein folding. Trends Biochem. Sci., 19, 331-336.

Fulton,A. (1982) How crowded is the cytoplasm? Cell, 30, 345-347.

Gilbert,H.F. (1997) Protein disulfide isomerase and assisted protein folding. J. Biol. Chem., 272, 29399-29402.

Goldberg,M.E., Rudolph,R. and Jaenicke,R. (1991) A kinetic study of the competition between renaturation and aggregation during refolding of denatured-reduced egg white lysozyme. Biochemistry, 30, 27902797.

Hartl,F.-U. (1996) Molecular chaperones in cellular protein folding. Nature, 381, 571-580.

Hillson,D.A., Lambert,N. and Freedman,R.B. (1984) Formation and isomerization of disulfide bonds in proteins: protein disulfideisomerase. Methods Enzymol., 107, 281-294.

Hwang,C., Sinskey,A.J. and Lodish,H.F. (1992) Oxidized redox state of glutathione in the endoplasmatic reticulum. Science, 257, 1496-1502.

Martin,J. and Hartl,F.-U. (1997) The effect of macromolecular crowding on chaperonin-mediated protein folding. Proc. Natl Acad. Sci. USA, 94, 1107-1112.

Minton,A.P. (1983) The effect of volume occupancy upon the thermodynamic activity of proteins: some biochemical consequences. Mol. Cell. Biochem., 55, 119-140.

Minton,A.P. (1992) Confinement as a determinant of macromolecular structure and reactivity. Biophys. J., 63, 1090-1100.

Minton,A.P., Colclasure,G.C. and Parker,J.C. (1992) Model for the role of macromolecular crowding in regulation of cellular volume. Proc. Natl Acad. Sci. USA, 89, 10504-10506.

Minton,K.W., Karmin,P., Hahn,G.M. and Minton,A.P. (1982) Nonspecific stabilisation of stress-susceptible proteins by stress-resistant proteins: a model for the biological role of heat shock proteins. Proc. Natl Acad. Sci. USA, 79, 7107-7111.

Puig,A. and Gilbert,H.F. (1994) Protein disulfide isomerase exhibits chaperone and anti-chaperone activity in the oxidative folding of lysozyme. J. Biol. Chem., 269, 7764-7771.

Puig,A., Lyles,M.M., Noiva,R. and Gilbert,H.F. (1994) The role of the thiol/disulfide centres and peptide binding site in the chaperone and anti-chaperone activities of protein disulfide isomerase. J. Biol. Chem., 269, 19128-19135.

Radford,S.E., Dobson,C.M. and Evans,P.A (1992) The folding of hen lysozyme involves partially structured intermediates and multiple pathways. Nature, 358, 302-307.

Roux,P., Delepierre,M., Goldberg,M.E. and Chaffotte,A.-F. (1997) Kinetics of secondary structure recovery during the refolding of reduced hen egg white lysozyme. J. Biol. Chem., 272, 24843-24849.

Song,J.-L. and Wang,C.-C. (1995) Chaperone-like activity of protein disulfide-isomerase in the refolding of rhodanese. Eur. J. Biochem., 231, 312-316

Swaminathan,R., Hwang,C.P. and Verkman,A.S. (1997) Photobleaching and anisotropy decay of green fluorescent protein GFP-S65T in solutions and cells: cytoplasmic viscosity probed by GFP translational and rotational diffusion. Biophys. J., 72, 1900-1907.

van den Berg,B., Chung,E.W., Robinson,C.V. and Dobson,C.M. (1999a) Characterisation of the dominant oxidative folding intermediate of hen lysozyme. J. Mol. Biol., 290, 781-796.

van den Berg,B., Robinson,C.V., Mateo,P.L. and Dobson,C.M. (1999b) The oxidative folding of hen lysozyme and its catalysis by protein disulfide isomerase. EMBO J., 18, 4794-4803.

Weissman,J.S. and Kim,P.S. (1991) Reexamination of the folding of BPTI: predominance of native intermediates. Science, 253, 1386-1393.

Zapun,A., Creighton,T.E., Rowling,P.J.E. and Freedman,R.B. (1992) Folding in vitro of bovine pancreatic trypsin inhibitor in the presence of proteins of the endoplasmic reticulum. Proteins, 14, 10-15.

Zimmerman,S.B. and Minton,A.P. (1993) Macromolecular crowding: biochemical, biophysical and physiological consequences. Annu. Rev. Biophys. Biomol. Struct., 22, 27-65.

Zimmerman,S.B. and Trach,S.O. (1991) Estimation of macromolecule concentrations and excluded volume effects for the cytoplasm of Escherichia coli. J. Mol. Biol., 222, 599-620.

Received September 15, 1999; revised and accepted October 25, 1999 